\title{
IMPORTÂNCIA DO ENVELHECIMENTO SAUDÁVEL COMO POLÍTICA PÚBLICA NO PÓS-PANDEMIA DA COVID-19
}

\author{
Iride Cristofoli Caberlon' \\ ORCID: 000-0002-8703-7038 \\ Letice Dalla Lana" \\ ORCID: 0000-0002-9624-8152
}

Maria Cristina Sant'Anna da Silva'

ORCID: 0000-0002-2714-4341

Lisiane Manganelli Girardi Paskulin"'

ORCID 0000-0003-1444-4086

Luiz Gustavo Fernandes da Rosa'v

ORCID 0000-0001-7509-0635

Marines Aires $v$

ORCID: 0000-0002-8257-2955

'Sociedade Brasileira de Geriatria e Gerontologia-RS, Porto Alegre, Rio Grande do Sul, Brasil.

"Universidade Federal do Pampa, Unipampa. Uruguaiana, Rio Grande do Sul, Brasil.

"'Universidade Federal do Rio Grande do Sul. Porto Alegre, Rio Grande do Sul, Brasil.

"V Universidade Luterana do Brasil.

Canoas, Rio Grande do Sul, Brasil.

vUniversidade Regional Integrada do Alto, Uruguai e das Missões. Frederico Westphalen, Rio Grande do Sul, Brasil.

Autor Correspondente: Iride Cristofoli Caberlon. Email: iridec27@gmail.com

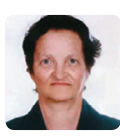

Como citar:

Caberlon IC, Lana LD, Silva MCS, Paskulin LMG, Rosa LGF, Aires M. Importância do Envelhecimento saudável como Política Pública no Pós-Pandemia da Covid-19. In: Santana RF (Org.). Enfermagem gerontológica no cuidado do idoso em tempos da COVID 19. Brasilia, DF: Editora ABen; 2021. 171 p.

(Serie Enfermagem e Pandemias, 5). https://doi.org/10.51234/aben.21.e05.c01

\section{INTRODUÇÃO}

Diferentes conceitos são utilizados para descrever o processo de envelhecimento humano, tais como os termos saudável e ativo. A expressão "envelhecimento saudável" remete à ideia de manutenção da capacidade funcional, ou seja, a associação entre a capacidade intrínseca do indivíduo, o ambiente e as interações entre sujeito e ambiente, mais do que propriamente a ausência de doenças ${ }^{(1)}$.

Com a pandemia da COVID-19, uma grande proporção de pessoas idosas no mundo, sobretudo no Brasil, está sendo influenciada a modificar comportamentos, visando à prevenção da doença. Sabe-se que essas pessoas são mais suscetíveis à pandemia da COVID-19 devido às alterações fisiológicas do processo de envelhecimento, associadas à lentificação da função imunológica e às alterações fisiopatológicas decorrentes de doenças crônicas e da fragilidade, aspectos provavelmente determinantes para o agravamento da infecção e para a maior mortalidade do segmento etário(2).

Em função da maior vulnerabilidade, houve a necessidade de manterem distanciamento social por período indefinido, provocando uma grande alteração nos seus hábitos de vida. Como impacto negativo, cita-se o rompimento de costumes que traziam sentido as suas vidas e que pode, por vezes, tornar-se um gatilho para sentimentos depres$\operatorname{sivos}^{(3)}$ e, também, levar à piora do estado geral da saúde.

Acrescido a isso, tem-se o fato de possíveis reduções no acompanhamento dos serviços de saúde e interrupção de tratamentos. Por outro lado, novos aprendizados, formas de autocuidado e de assistência às pessoas idosas têm sido implementados, o que pode colaborar para a ampliação das fronteiras do envelhecimento ativo. Nessa perspectiva, a Enfermagem tem papel fundamental tanto ao advogar pela saúde, participação e segurança dessas pessoas neste novo cenário, como ao ofertar novas formas de cuidado que têm sido experienciadas na vigência da pandemia e que podem beneficiá-las hoje e amanhã. 


\section{OBJETIVO}

Refletir sobre a importância do envelhecimento saudável como política pública de cuidado à população no pós-pandemia da COVID-19, indicando desafios e legados.

\section{MÉTODOS}

Este estudo teórico-reflexivo parte das políticas públicas voltadas ao envelhecimento ativo e, a seguir, explora desafios e avanços no cuidado contínuo e humanizado as pessoas idosas e os legados após a pandemia da COVID-19. Para subsidiar a reflexão, utilizaram-se políticas públicas internacionais e nacionais e artigos pertinentes à temática. A reflexão abordou dois temas: envelhecimento ativo e políticas públicas relacionadas;desafios e legados no pós-pandemia na perspectiva do envelhecimento saudável.

\section{RESULTADOS E DISCUSSÃO}

No sentido de organizar os resultados oriundos desta reflexão, apresentam-se os temas envelhecimento ativo, saudável e políticas públicas relacionadas, seguidos de desafios e legados no pós- pandemia na perspectiva do envelhecimento ativo. Tais temas aqui apresentados separadamente complementam-se e articulam-se ao cuidado da pessoa idosa na Rede de Atenção à Saúde (RAS).

\section{ENVELHECIMENTO E POLÍTICAS PÚBLICAS RELACIONADAS}

Desde 2005, a OMS instituiu a Política do Envelhecimento Ativo com o objetivo de aumentar a expectativa de vida e promover melhor qualidade de vida à população. Para otimizar o envelhecimento ativo, as pessoas devem perceber e desenvolver o seu potencial para o bem-estar físico, social e mental;ter a possibilidade de aprendizagem formal e informal, oficinas, cursos livres, atividades educativas gerais;participar da sociedade de acordo com suas necessidades, desejos e capacidades;ter segurança, proteção e cuidados adequados, incluindo transportes acessíveis, segurança econômica, condições de habitação, convívio familiar e comunitário, proteção judiciária(4).

Para a elaboração de políticas e programas exitosos visando ao envelhecimento ativo é preciso levar em consideração seus fatores determinantes, aspectos da realidade situacional, econômicos, sociais, ambientais, pessoais, comportamentais, de serviços sociais e de saúde. Tais fatores compreendem os componentes transversais, gênero e cultura;os relacionados aos sistemas de saúde e serviço social, promoção da saúde e prevenção de doenças, serviços curativos, assistência em longo prazo, serviços de saúde mental;aos fatores comportamentais, atividade física, alimentação saudável, abstinência de fumo e de álcool, saúde oral, uso de medicamentos e acesso apropriado;os relacionados a aspectos pessoais, biologia e genética, fatores psicológicos (incluem inteligência e capacidade cognitiva);os relacionados ao ambiente físico, moradia segura, água potável, ar puro, alimentos seguros e adequados;os relacionados ao ambiente social, apoio social, violência e maus-tratos, educação e alfabetização;os fatores econômicos, trabalho, renda, proteção social(4).

Incluindo os referenciais do envelhecimento ativo, em 2009, a Organização Pan-Americana da Saúde (OPAS) estabeleceu o Plano de Ação sobre a Saúde das Pessoas Idosas. O foco foi promover a longevidade saudável e o bem-estar por meio de estratégias e ações participativas e eficazes para cumprir os seguintes compromissos: saúde das pessoas idosas nas políticas públicas;adequação dos sistemas de saúde para enfrentar os desafios associados ao envelhecimento da população e formação de recursos humanos ${ }^{(5)}$.

No Brasil, a Política Nacional de Saúde da Pessoa Idosa (PNSPI), de 2006, incluiu a Promoção do Envelhecimento Ativo e Saudável entre suas diretrizes ${ }^{(6)}$. A PNSPI destaca que o principal problema que pode afetar a 
pessoa idosa é a perda de sua capacidade funcional, isto é, a perda das habilidades físicas e mentais necessárias para a realização de atividades da vida diária. Ao aproximar tal política dos pilares do Envelhecimento Ativo, verifica-se que ela igualmente destaca a importância da promoção da autonomia e da independência dos indivíduos idosos, direcionando medidas coletivas e individuais de saúde, a fim de promover essa forma de envelhecimento.

Também a Política Nacional da Promoção da Saúde, de 2014, guarda consonância com as diretrizes da Política do Envelhecimento Ativo ao estabelecer ações prioritárias a serem adotadas ao longo da vida, como alimentação saudável, prática corporal/atividade física, prevenção e controle do tabagismo, do uso de álcool e de outras drogas, redução da morbimortalidade, das causas externas e prevenção da violência(7).

Para atender a essa política, é preciso identificar as necessidades de saúde das pessoas idosas considerando sua capacidade funcional ou condição de funcionalidade, importante indicador de saúde dessa população. Para atender a essas necessidades é preciso estabelecer uma linha de cuidado efetiva. Nessa perspectiva, em 2018, instituiu-se o documento com orientações técnicas aos estados, ao Distrito Federal e aos municípios para nortear a construção e a implementação dessa linha, visando à atenção integral das pessoas idosas na RAS(8).

Para tal, o Decreto no 9.921, de 18 de julho de 2019, estabelece as competências ampliadas na implementação da PNSPI. Assim, no que tange ao Ministério da Saúde, por meio da Secretaria de Atenção Primária à Saúde, compete, dentre outras ações, desenvolver e apoiar programas de prevenção, de educação e de promoção à saúde da pessoa idosa, visando a estimular a permanência dessas pessoas na comunidade, desempenhando papel social ativo, com autonomia e independência, estimular a criação de hospital-dia, de centro-dia, de unidades de atendimento domiciliar e de outros serviços alternativos à pessoa idosa ${ }^{(9)}$.

Além do setor de saúde, é preciso destacar que, para a promoção do envelhecimento ativo, a OMS recomenda a participação de todas as políticas setoriais, intersetoriais das esferas de governo, as quais precisam garantir acesso ao lazer, ao esporte, à cultura, à habitação, ao emprego, à renda, dentre outros fatores ${ }^{(10)}$.

\section{DESAFIOS E LEGADOS NO CUIDADO APÓS A PANDEMIA NA PERSPECTIVA DO ENVELHECIMENTO ATIVO}

Um dos grandes legados dessa pandemia às pessoas idosas parece ser os novos aprendizados. Para manter contato social e atender às suas necessidades, esse grupo etário precisou aprender rapidamente a utilizar os recursos tecnológicos e de informação para manter contatos com familiares e amigos, evitando a solidão.

O uso das tecnologias também tem permitido a manutenção da autonomia do referido grupo, ao decidirem, a partir dos seus domicílios, como se relacionar com atividades do dia a dia, com destaque para o uso de serviços bancários e a realização de compras sem depender de seus familiares ou outras pessoas.

Além disso, o uso das redes sociais oportunizou adquirir novas práticas mediante a troca de mensagens sobre diferentes temas, como receitas de culinária, informações sobre saúde, desafios para a prática de atividade física no domicílio, entre outras. Contudo, segue sendo um grande desafio para muitas pessoas a utilização das tecnologias digitais para a manutenção dos vínculos e à realização das atividades.

Durante a pandemia, profissionais de saúde também têm se utilizado das redes sociais, de aplicativos e de videoconferências para fornecer orientações à população. Tais práticas vêm contribuindo para o estímulo e manutenção de atividades físicas no domicílio, de atividades de promoção da saúde mental, de lazer (músicas, jogos, leituras, desafios de memória, entre outras), de práticas religiosas, de orientações sobre prevenção de quedas, do uso correto de medicações no tratamento de doenças crônicas, além de divulgar parâmetros para situações que indicam a necessidade de busca por ajuda presencial.

Outro legado, decorrente das medidas impostas para o controle da disseminação da doença, diz respeito à reorganização do acesso e da assistência à saúde da população idosa. Na RAS, enfermeiros e médicos buscam adotar, em sua prática, a Política da TelesSaúde, que foi instituída pelo Ministério da Saúde ${ }^{(11)}$, por meio do telemonitoramento e da teleconsulta. O telemonitoramento permite acompanhar a situação de saúde das pessoas idosas durante o isolamento, enquanto que a teleconsulta, para agravos à saúde, tem 
sido implementada, oportunizando avaliar, orientar, propor medidas de prevenção, reabilitação de agravos e promoção à saúde desse segmento.

O uso de tecnologias de cuidado remoto deve ser aplicado principalmente em portadores de condições crônicas, visando à garantia do envelhecimento ativo a tal parcela da população. Todavia, a implementação da TelesSaúde, em sua integridade, pode ser um desafio para os serviços diante da dificuldade de acesso digital das pessoas idosas, sendo preciso considerar equidade e acessibilidade para o seu uso. Conforme evidenciado em estudo de revisão integrativa sobre o tema, nem todos os usuários são beneficiados, principalmente os mais vulneráveis e os que têm dificuldade na utilização da tecnologia ${ }^{(12)}$.

Na pós-pandemia, para a ampliação dessas estratégias e para que elas possam fazer parte do cotidiano dos serviços de saúde, dois dos desafios serão aumentar os recursos tecnológicos e contemplar as áreas de difícil acesso digital. Assim, urge que os órgãos federais reformulem as diretrizes operacionais do setor Saúde ao segmento idoso, disponibilizando sistemas integrados que permitam acompanhá-lo nos diferentes pontos de atenção e que evoluam também nas formas de manter contato com eles, como a utilização de aplicativos que possibilitam a visualização do agendamento de atendimentos e resultados de exames.

Nessa reestruturação, é preciso ampliar ainda a qualificação dos recursos humanos para atender ao crescente contingente de pessoas idosas na população. Vale destacar que promover a formação e educação permanente dos profissionais de saúde, visando a qualificar a assistência às pessoas idosas no contexto de promoção, de prevenção, de avaliação, de tratamento, de reabilitação e de cuidados paliativos é uma das diretrizes da Política Nacional da Pessoa Idosa ${ }^{(6)}$.

Não obstante, cada indivíduo precisa gerar oportunidades para proteger sua vida e saúde de forma contínua, exercendo sua autonomia e sendo protagonista de seus atos. Entre essas oportunidades, está a participação em ações educativas de saúde em novos formatos, nos quais destaca-se o profissional enfermeiro, que deve estimular a escolha de comportamentos promotores de saúde e orientar como adotá-los, a fim de manter a qualidade de vida e evitar o declínio funcional desse indivíduo.

Novos formatos para o atendimento de pessoas idosas que possuem capacidade funcional reduzida têm sido utilizados e poderão ser um legado para o novo contexto de vida. Quando há perda parcial ou total da autonomia e da independência, surge a necessidade de receberem cuidados intermitentes ou permanentes no domicílio.

Nesse cenário, o cuidado é realizado predominantemente por familiares e por cuidadores informais, muitos deles despreparados para assumir esse papel. Além das orientações sobre cuidados com as atividades básicas da vida diária, que já têm sido veiculadas, cartilhas com outras orientações, informações e ilustrações foram disponibilizadas para os cuidadores, visando à prevenção do contágio, à promoção da saúde e à proteção aos direitos sociais, tanto de quem é cuidado, como de quem cuida.

De forma semelhante, os cuidados fornecidos pelos familiares dessas pessoas, inclusive aos acamados, vêm sendo sustentados a distância por meio de avaliação multidimensional e orientações profissionais realizadas com o apoio do telemonitoramento, nos diversos níveis de atenção. Mesmo em um novo formato, as orientações devem seguir enfocando a capacidade de tomar decisões e a manutenção da independência funcional para a realização das atividades do cotidiano.

Em decorrência da pandemia, a suspensão das atividades presenciais dos grupos de apoio às pessoas idosas pode ter impactado na saúde física e mental destas. Possivelmente, após o controle da doença, por meio da disponibilização da vacina específica ao vírus, muitas ainda sentirão medo e receio de retomá-las. Nesse caso, caberá aos profissionais de saúde encorajá-las a retomada gradual dessas atividades, buscando novas formas de participação no contexto comunitário e nos serviços de saúde.

Importante comentar, ainda, a contradição entre o estímulo à campanha de vacinação anti-influenza e à importância do distanciamento controlado durante a pandemia, o que obrigou a reconfiguração da operacionalização da campanha. Assim, uma das estratégias que passou a ser adotada foi a intitulada drive-thru, que proporcionou às pessoas idosas, principalmente as com maior dificuldade de mobilização, receberem a vacina 
dentro de seus carros. Essa estratégia poderá ser implementada, seja para a vacinação contra a COVID-19, seja para a prevenção de outras doenças. Outra estratégia de grande factibilidade, nem sempre adotada, mas já utilizada em municípios de pequeno porte, que também deveria ser efetivamente implementada, é a imunização domiciliar agendada, que contempla grande parcela da população idosa mais dependente.

Apesar dos avanços em termos de organizações no sistema de saúde para atender às necessidades de saúde das pessoas idosas, ainda permanecem as questões socioeconômicas, as desigualdades locais, regionais, estaduais, as síndromes geriátricas, a tripla carga de doenças, os cuidados no domicílio e a violência. Nesse período de distanciamento social, especialmente para as pessoas que são vulneráveis e frágeis, os diversos tipos de violência, física, psicológica, negligência, suborno financeiro e econômico, dentre outras, tendem a aumentar porque geralmente o agressor é um morador da mesma casa ou membro da família. Esses aspectos seguirão sendo desafios a serem superados em um contexto tão diverso como o do Brasil.

As novas estratégias aqui relatadas, entre outras, servirão para ampliar as ações programáticas voltadas ao cuidado integral com os indivíduos idosos. Na perspectiva da enfermagem, essas novas estratégias também têm impacto ao fortalecer a atuação dos profissionais e ao promover o cuidado à pessoa idosa e sua família com dignidade, segurança, autonomia e independência.

\section{CONSIDERAÇÕES FINAIS}

Os principais legados da pandemia da COVID-19, na perspectiva dos autores deste estudo de reflexão, envolvem diferentes formas de organização e comunicação entre redes de apoio formais e informais. Quanto aos desafios abordados, que já fazem parte do contexto de vida brasileiro e que seguirão presentes, estão as desigualdades sociais, as síndromes geriátricas, a tripla carga de doenças, as situações de violência, a integralidade do cuidado, e acrescenta-se a implementação da TelesSaúde na Rede de Atenção à Saúde.

As reflexões deste estudo denotam que a Política do Envelhecimento Saudável é um investimento em longo prazo, importante para proporcionar que as pessoas mantenham a capacidade funcional pelo maior tempo possível. Na pandemia, novas estratégias de cuidado e de participação de gestores públicos, profissionais, pessoas idosas e suas famílias foram utilizadas, demonstrando que os programas e as ações de saúde podem e precisam ser ampliados.

\section{AGRADECIMENTO}

\section{Ao Departamento Científico de Enfermagem Gerontológica da ABEn Nacional.}

\section{REFERÊNCIAS}

1. Beard JR, Officer A, Carvalho IA, Sadana R, Pot AM, Michel JP, et al. The World Report on ageing and health: a policy framework for healthy ageing. Lancet. 2016;387(10033):2145-54. https://doi.org/10.1016/S0140-6736(15)00516-4

2. Venturini L, Kinalski SS, Benetti ERR. Aspectos gerontológicos do cuidado critico às pessoas idosas com covid-19. In: Santana RF. Enfermagem gerontologica no cuidado do idoso em tempo da COVID 19. Brasilia, DF: Editora ABEn; 2020. p.5863. (Serie Enfermagem e Pandemias, 1). https://doi.org/10.51234/aben.20.e01.c09

3. Hammerschmidt KSA, Santana RF. Saúde do idoso em tempos de pandemia Covid-19. Cogitare Enferm[Internet]. 2020 [cited 2020 Jun 07];25:e72849. Available from: https://pesquisa.bvsalud.org/portal/resource/pt/biblio-1095404

4. World Health Organization (WHO). Envelhecimento ativo: uma política de saúde [Internet]. Brasília: Organização Pan-Americana da Saúde, 2005[cited 2020 Jun 07]. Available from: https://bvsms.saude.gov.br/bvs/publicacoes/ envelhecimento_ativo.pdf

5. Organização Pan-Americana da Saúde (OPAS). Plan de acción sobre la salud de las personas mayores incluyendo el envejecimiento activo y saludable [Internet]. 2009[cited 2020 Jun 07]. Available from: https://iris.paho.org/bitstream/ handle/10665.2/33934/CD49-08-s.pdf?sequence=4\&isAllowed=y 
6. Ministério da Saúde (BR). Política Nacional de Saúde da Pessoa Idosa. Portaria GAB/MS n².528, de 19 de outubro de 2006 [Internet]. Brasília: Ministério da Saúde; 2006[cited 2020 Jun 07]. Available from: https://bvsms.saude.gov.br/bvs/ saudelegis/gm/2006/prt2528_19_10_2006.html

7. Ministério da Saúde (BR). Política Nacional de Promoção da Saúde: PNaPS: revisão da Portaria MS/GM n 687, de 30 de março de 2006 [Internet]. Brasília: Ministério da Saúde, 2014[cited 2020 Jun 07]. Available from: http://bvsms.saude.gov.br/ bvs/publicacoes/pnps_revisao_portaria_687.pdf

8. Ministério da Saúde (BR). Orientações técnicas para a implementação de Linha de Cuidado para Atenção Integral à Saúde da Pessoa Idosa no Sistema Único de Saúde - SUS [Internet]. Brasília: Ministério da Saúde, 2018 [cited 2020 Jun 07]. Available from: https://bvsms.saude.gov.br/bvs/publicacoes/linha_cuidado_atencao_pessoa_idosa.pdf

9. Presidência da República (BR). Decreto № 9.921 de 18 de julho de 2019, que consolida atos normativos editados pelo poder executivo federal que dispõem sobre a temática da pessoa idosa [Internet]. Brasília: Distrito Federal, 2019 [cited 2020 Jun 07]. Available from: http://www.planalto.gov.br/ccivil_03/_Ato2019-2022/2019/Decreto/D9921.htm\#art48

10. Organização Mundial da Saúde. Resumo relatório mundial de envelhecimento e saúde. Genebra: OMS, 2015. Available from: https://apps.who.int/iris/bitstream/handle/10665/186468/WHO_FWC_ALC_15.01_por.pdf?sequence=6

11. Ministério da Saúde (BR). Portaria n².546, de 27 de outubro de 2011 [Internet]. 2011 [cited 2020 Jun 07]. Redefine e amplia o Programa Telessaúde Brasil, que passa a ser denominado Programa Nacional Telessaúde Brasil Redes (Telessaúde Brasil Redes). Available from: http://bvsms.saude.gov.br/bvs/saudelegis/gm/2011/prt2546_27_10_2011.html

12. Catapan SC, Calvo MCM. Teleconsulta: uma revisão integrativa da interação médico-paciente mediada pela tecnologia. Rev Bras Educ Med. 2020;44(1):e002. https://doi.org/https://doi.org/10.1590/1981-5271v44.1-20190224 